\begin{tabular}{|l|l|l|l|} 
Eiszeitalter und Gegenwart & Band 13 & Seite 129-137 & Öbringen/Württ., 1. September 1962 \\
\hline
\end{tabular}

\title{
Weitere Funde pleistozäner Molluskenfaunen bei München
}

\author{
Von Margrit Brunnacker und Karl Brunnacker, München
}

Mit 4 Tabellen im Text

\begin{abstract}
$\mathrm{Z} u$ s a m m e $\mathrm{f}$ a s s u $\mathrm{g}$ : Von fünf Stellen wird eine unter rißeiszeitlichem Hochterrassenschotter gefundene interglaziale Molluskenfauna beschrieben (s. Tab. 1); sie wird ökologisch gedeutet und stratigraphisch mit anderen Molluskenfaunen verglichen (s. Tab. 2); ferner wird die Genese des einbettenden Sedimentes erörtert (s. Tab. 3). Die sich daraus ergebenden Gesichtspunkte für eine Abtrennung des ausgehenden Mindel/Riß-Interglazials von der (beginnenden?) Rißeiszeit werden diskutiert (s. Tab. 4); demnach deutet sich wenigstens für einen Teil der Profile eine Sedimentationslücke zwischen dem molluskenführenden interglazialen Horizont und dem darüberliegenden fluvioglazialen Schotter der Rißeiszeit an.
\end{abstract}

A bstract: A fauna of interglazial molluscs is described (tab. 1) which, in five sites, was found underneath a gravel terrace of Rissian age. It is interpreted ecologically, and compared stratigraphically with other faunas (tab. 2). The genesis of the embedding sediment is also treated (tab. 3). The results of these studies are discussed with respect to the possibility of separating stratigraphically the declining Mindel/Riß interglacial age from the (beginning of the ? Riß glacial stage (tab. 4). The existence of a stratigraphical break is indicated in at least some of the profiles studied, between the interglacial horizon with molluscs and the overlying fluvioglacial gravels of the Riß stage.

Durch R. SCHRÖDER (1915) wurden die bis dahin bekanntgewordenen Funde pleistozäner Molluskenfaunen im Hochterrassenschotter des Münchener Gebietes umfassend dargestellt. Erst H. Nathan (1953) hat ein weiteres, in der Ausbildung jedoch abweichendes Vorkommen südwestlich Moosburg beschrieben. Bei Untersuchung der Hochterrassenreste östlich der Isar wurden in den letzten Jahren schließlich die hier behandelten Molluskenfaunen gefunden. Auf die Ausbildung der Gesamtprofile und die genaue Lage der Profile wird in einem späteren, schotterpetrographischen Beitrag eingegangen werden.

\section{Die Fauna (Margrit Brunnacker)}

Die von R. SchröDer (1915) bearbeiteten Fundpunkte in den Kiesgruben der Hochterrasse bei Oberföhring und Ismaning sind heute nicht mehr zugänglich. Jedoch wurden bei von M. KunsCH durchgeführten geologischen Untersuchungen auf Gradabteilungsblatt Ismaning Feinsedimente mit Molluskenfaunen gefunden, die durch Hochterrassenschotter überlagert werden, und zwar im Bereich der kleinen Hochterrasseninsel von Eicherloh (Aufschluß-Nr. X) und Eichenried (Nr. IX), ferner auf Gradabteilungsblatt Moosburg im Gebiet des durch H. Nathan (1953) genannten „Hochterrassen“-Restes mit Fagotia acicularis FÉR. bei Niederhummel (Nr. II) und Thonstetten (Nr. VII und Nr. VIII).

Die Zusammensetzung dieser Faunen (Tab. 1) zeigt in Eicherloh, Eichenried und Niederhummel große Ähnlichkeit mit derjenigen, die R. SCHröDER (1915) als Interglazialfauna aus der Umgebung von Ismaning beschrieben hat. Man darf weiter annehmen, daß diese Fauna bereits in die Zeit etwas nach dem interglazialen Klimaoptimum gehört; denn es handelt sich um eine Laubwald- evtl. auch Mischwaldfauna, wie sie rezent südlich München bzw. im weiteren Alpenvorland gefunden werden könnte. Bemerkenswert ist darin der bislang von hier fossil nicht bekannte Goniodiscus perspectivus MEG., der heute südlich München in feuchteren Laubwäldern vorkommt. Die Ähnlichkeit mit der genannten, durch R. ScHRöDER (1915) bearbeiteten Fauna zeigt sich auch darin, daß es sich praktisch um keine Mischfaunen verschiedener Biotope handelt, wie es normalerweise bei Einbettung in Flußablagerungen der Fall ist. Deshalb dürfte auch die Durchsicht auf Ostracoden erfolglos geblieben sein. Wesentlich geringer ist hingegen die Artenzahl der Mollusken im Profil Thonstetten Nr. VII und VIII. Doch ist auch hier die Sedimentausbil- 
Tabelle 1

Verzeichnis der gefundenen Molluskenarten

\begin{tabular}{|c|c|c|c|c|c|c|}
\hline & 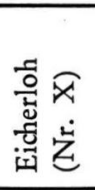 & 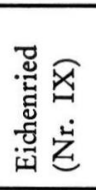 & 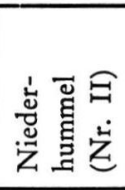 & 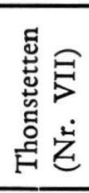 & 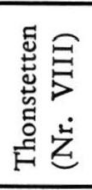 & 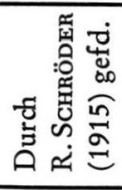 \\
\hline $\begin{array}{c}\text { Succinea pfeifferi Rossm. } \\
\text { " oblonga Drap. }\end{array}$ & $\mathrm{h}$ & & $\begin{array}{l}\mathrm{e} \\
\text { sh }\end{array}$ & & & + \\
\hline Cochlicopa lubrica MüLL. & $\mathrm{h}$ & sh & $\operatorname{sh}$ & $\mathbf{h}$ & & + \\
\hline Pupilla sp. & & & & e & & \\
\hline Pagodulina pagodula Desm. & & e & & & & \\
\hline Vallonia pulchella MüLL. & $\mathrm{h}$ & e & e & e & & + \\
\hline$" \quad$ costata MüLL. & & & & $\mathrm{e}$ & $\mathrm{h}$ & + \\
\hline Cochlodina sp. & & & e & & e & \\
\hline Punctum pygmaeum Drap. & & e & & & & \\
\hline Goniodiscus rotundatus MüLL. & $\mathrm{h}$ & sh & sh & & & + \\
\hline " perspectivus MEG. & & sh & & sh & & \\
\hline Retinella radiatula ALDER & $\mathrm{h}$ & & $\mathrm{h}$ & & & \\
\hline " nitidula Drap. & & sh & & & & + \\
\hline $\begin{array}{ll}\Rightarrow & \text { nitens Mich. } \\
& \text { sp. }\end{array}$ & & e & & sh & sh & \\
\hline Vitrea diaphana Drap. & & $\mathrm{h}$ & & & & \\
\hline $\begin{array}{l}\text { crystallina MüLL. } \\
\text { sp. }\end{array}$ & & $\mathrm{h}$ & & & $\mathrm{h}$ & + \\
\hline Euconulus trochiformis MONT. & $\mathrm{h}$ & $\mathrm{h}$ & & & & + \\
\hline Daudebardia brevipes DRAP. & & e & & & & \\
\hline Semilimax semilimax FÉR. & e & & e & & & + \\
\hline Fruticicola sericea DRAP. & $\mathrm{sh}$ & & & & & + \\
\hline " unidentata DraP. & & e & & & & + \\
\hline " edentula Drap. & & e & & & & + \\
\hline Helicodonta obvoluta MüLL. & & $\mathrm{h}$ & & & & \\
\hline Arianta arbustorum L. & & h & e & e & & + \\
\hline Cepaea sp. & & & e & e & & \\
\hline Carychium tridentatum Risso & & & sh & & & + \\
\hline Galba truncatula MüLL. & & e & $\mathrm{sh}$ & & & \\
\hline Gyraulus albus MüLL. & & & $\mathrm{e}$ & & & \\
\hline " laevis ALDER & & & $\mathrm{h}$ & & & \\
\hline Acme polita HARTM. & & & $\mathrm{e}$ & & & + \\
\hline
\end{tabular}

e $=$ einzeln (1-2 Gehäuse)

$\mathrm{h}=$ häufig (3-6 Gehäuse)

sh $=$ sehr häufig (mehr als 6 Gehäuse)

in jeweils rd. $5 \mathrm{~kg}$ Sediment 


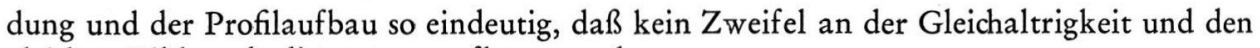
gleichen Bildungsbedingungen aufkommen kann.

R. SCHRöDER (1915) hat in diesem interglazialen Fundhorizont insgesamt 26 Arten festgestellt, von denen 15 auch in den neuen Aufsammlungen gefunden werden konnten. Infolge der regen Kiesentnahme sind die hier aufgeführten neuen Fundstellen zum Teil bereits wieder weitgehend abgebaut, so daß auch durch Aufsammlungen über einen längeren Zeitraum hinweg die Ausbeute kaum zu erhöhen ist.

Mischfaunen finden sich ebenfalls im Hochterrassenschotter, und zwar in einem etwas höheren stratigraphischen Niveau (R. SCHRöDER 1915), ferner in eindeutig interglazialen Ablagerungen südwestlich Moosburg (H. Nathan 1953).

Damit lassen sich im Bereich der mittel- und jungpleistozänen Ablagerungen des Münchener Gebietes, ergänzt durch einige andere Funde, folgende Faunen verschiedener $\mathrm{Zu}$ sammensetzung, Lagerung und stratigraphischer Situation unterscheiden:

1. An der Basis der Hochterrasse in zum Teil in Schollen aufgearbeiteten Feinsedimenten eine interglaziale Fauna terrestrer Biotope; hierzu gehört die von R. SchröDER (1915) beschriebene Fauna und die jetzt neu hinzukommenden Funde.

2. Eine ebenfalls von R. SCHRÖDER (1915) beschriebene, in den Hochterrassenschotter, jedoch in etwas höherem Niveau eingelagerte, mit fast 40 Arten verhältnismäßig reiche Mischung von Land- und Wassermollusken mit kühleren bis kalten Klimaansprüchen (=„Jüngere Fauna“). Bezeichnenderweise wurden hier bereits Reste des Halsbandlemmings gefunden.

3. Bei Lochhausen wurde ferner im Hochterrassenschotter eine Fauna (4 Arten) mit Dominanz der Succineen festgestellt. Sie wird von R. SchröDER (1915) der unter 2. genannten Fauna zeitlich gleichgesetzt. Diese Gleichsetzung scheint aber insofern nicht eindeutig zu sein, als diese Fauna bereits eine dem (würmeiszeitlichen) Löß Südbayerns recht ähnliche artenarme Zusammensetzung aufweist (vgl. M. Brunnacker \& K. BrunNacker 1956). Allerdings kann im Löß des Tertiärhügellandes in S-Exposition noch Arianta arbustorum L. hinzukommen. Diese Fauna, gleich ob im würmzeitlichen Löß oder im rißeiszeitlichen Hochterrassenschotter, zeigt auf relativ feuchte kaltklimatische Verhältnisse. Hingegen deutet eine andere Fauna mit Succinea oblonga DRAP., Pupilla muscorum L., Fruticicola striata MüLL. und Vallonia tenuilabris A. BR. in dem einem alten Schotter (Mindel) eingelagerten Lehm bei Riedlingen auf trockenere glaziale Verhältnisse. Hierüber wird durch H. Graul in einer eigenen Arbeit berichtet werden.

4. Die sicher jüngere als die erste genannte interglaziale Fauna südwestlich Moosburg liegt hingegen als Mischfauna mit Fagotia acicularis FÉR. im Schotter bzw. in darin vorkommenden sandigeren Lagen - hier zeigt sich in der Art des einbetenden Sedimentes eine abweichende Genese an.

5. In einem Auemergel über diesem Fagotienschotter kommt neben einigen anderen, durch Einzelexemplare vertretene Arten vor allem Gyraulus gredleri GRDL. vor. Verhältnismäßig kühle Klimaverhältnisse scheinen hier angedeutet, ebenso wie in der etwas artenreicheren Fauna der "Seekreide“ von Hörmating (vgl. R. DЕнм in E. EBERs 1960).

Für die aufgezählten mittel- bis jungpleistozänen, in fluviatilen Feinsedimenten, bzw. ausnahmsweise auch in Schotter, vorkommenden Molluskenfaunen ergibt sich damit die in Tab. 2 dargelegte, vereinfachte Gliederung nach ökologischen Ansprüchen unter Berücksichtigung der stratigraphischen Stellung. Auch weitere, bereits veröffenlichte Faunen (R. SChröper 1915, J. SChröDer \& R. Dehm 1951) sowie inzwischen neu hinzugekommene, noch nicht publizierte Vorkommen des Alpenvorlandes können in ein derartiges Schema eingebaut werden.

Die in Tab. 2 angeführten Mischfaunen lassen sich normalerweise als aus verschiedensten Biotopen zusammengeschwemmt erklären. Daneben gibt es aber auch auf Sukzessionen 


\section{Tabelle 2}

Die mittel- und jungpleistozänen Molluskenfaunen bei München (durch einige andere Vorkommen ergänzt), gegliedert nach zeitlicher Stellung und ökologischer Bewertung

\begin{tabular}{|c|c|c|c|c|c|}
\hline $\begin{array}{l}\text { Klimatische } \\
\text { Bewertung }\end{array}$ & Landfaunen & Wasserfaunen & Mischfaunen & $\begin{array}{l}\text { Einbettendes } \\
\text { Sediment }\end{array}$ & $\begin{array}{l}\text { Zeitliche } \\
\text { Stellung }\end{array}$ \\
\hline $\begin{array}{l}\text { kalt, } \\
\text { rel. feucht }\end{array}$ & $\begin{array}{l}\text { Lößfaunen mit } \\
\text { Dominanz der } \\
\text { Succineen }\end{array}$ & & & Löß & Würmeiszeit \\
\hline kühl bis kalt ? & & $\begin{array}{l}\text { Auemergelfauna von } \\
\text { Oberhummel }\end{array}$ & & Auemergel & $\begin{array}{l}\text { Ende des Riß/Würm- } \\
\text { Interglazials } \\
\text { oder Beginn der } \\
\text { Würmeiszeit }\end{array}$ \\
\hline $\begin{array}{l}\text { rel. } \\
\text { sommerwarm }\end{array}$ & & & $\begin{array}{l}\text { Fagotia-Fauna } \\
\text { SW Moosburg }\end{array}$ & Schotter & $\begin{array}{l}\text { Riß/Würm-Inter- } \\
\text { glazial }\end{array}$ \\
\hline kühl bis kalt & $\begin{array}{l}\text { Succineenfauna } \\
\text { im Hochterrassen- } \\
\text { schotter bei } \\
\text { Lochhausen }\end{array}$ & & $\begin{array}{l}\text { "Jüngere" Fauna im } \\
\text { Hochterrassen- } \\
\text { schotter } \\
\text { NO München }\end{array}$ & $\begin{array}{l}\text { Feinsediment } \\
\text { zwischen Schotter }\end{array}$ & $\begin{array}{l}\text { Beginn ? der } \\
\text { Rißeiszeit }\end{array}$ \\
\hline $\begin{array}{l}\text { etwa wie heute } \\
\text { (warm, feucht) }\end{array}$ & $\begin{array}{l}\text { Laubwaldfauna an } \\
\text { der Basis des } \\
\text { Hochterrassen- } \\
\text { schotters } \\
\text { NO München }\end{array}$ & & & $\begin{array}{l}\text { Feinsediment nächst } \\
\text { der Basis des Hoch- } \\
\text { terrassenschotters }\end{array}$ & $\begin{array}{l}\text { Mindel/Riß- } \\
\text { Interglazial }\end{array}$ \\
\hline $\begin{array}{l}\text { kalt, } \\
\text { rel. trocken }\end{array}$ & $\begin{array}{l}\text { „Löß“-Fauna } \\
\text { von Riedlingen }\end{array}$ & & & $\begin{array}{l}\text { Feinsediment } \\
\text { zwischen Schotter }\end{array}$ & Mindeleiszeit \\
\hline
\end{tabular}


beruhende Mischfaunen infolge von Verlandung oder Ansteigen bzw. Absinken eines Grundwasserspiegels oder infolge klimatischer Anderungen. Doch ist es in den meisten Fällen kaum möglich, die Ursache für das Zustandekommen einer derartigen Mischfauna genauer zu erfassen. Deshalb ist z. B, auch der Einzelfund von Galba truncatula MülL. im Profil Eichenried und das recht häufige Vorkommen in Niederhummel vorläufig ohne größere Bedeutung. Möglicherweise ist auch das etwas gehäufte Vorkommen von Succinea pfeifferi Rossm. und Vallonia pulchella MüLl. in Eicherloh bzw. von Succinea oblonga Drap. in Niederhummel durch eine Sukzession erklärbar, und auch die oben unter 2. genannte "Jüngere Fauna " aus dem Hochterrassenschotter könnte vielleicht $z$. T. darauf zurückgehen.

Abgesehen von einigen Neufunden von Mollusken und den neuen Fundstellen wären diese Beobachtungen beim derzeitigen Forschungsstand über die pleistozänen Erosionsund Akkumulationsphasen ohne größere Bedeutung, würde hier nicht die Möglichkeit bestehen, die Beziehungen zum Einbettungsmittel zu untersuchen. Außerdem ist die Feststellung möglich, daß diese Fauna um ein Interglazial älter ist als der Fagotienschotter südwestlich Moosburg; denn in Niederhummel lagert zwischen beiden molluskenführenden Schichten ein eiszeitlicher Schotter.

\section{Das einbettende Sediment (Karl Brunnacker)}

Nach den Feststellungen von R. SCHRöDer (1915) liegen die interglazialen Molluskenfaunen im Nordosten Münchens in umgelagerten Schollen von Feinsedimenten nächst der Basis des Hochterrassenschotters. Dies ist auch in Niederhummel der Fall. Bei den übrigen Profilen hat sich folgendes gezeigt: Die Feinsedimente liegen in Thonstetten Nr. VII zumindest teilweise in situ dem Tertiär unmittelbar auf. In Thonstetten Nr. VIII sind sie zum Teil in Schollen aufgearbeitet und verhältnismäßig stark sandig ausgebildet (und liefern deshalb eine Fauna in schlechtem Erhaltungszustand); sie lagern hier einem an Tertiärmaterial reichen Quartärschotter des Ampertales auf. Darüber folgt in Thonstetten Nr. VII Kalkschotter und in Thonstetten Nr. VIII ein wiederum an Tertiärmaterial sehr reicher Schotter. Dieser hangende Schotter ist an beiden Stellen leicht „vermorscht". In Niederhummel handelt es sich um eine kleine aufgearbeitete Scholle, die zwischen umgelagertem Tertiärmaterial im Liegenden und etwas morschem kalkgeröllreichem Schotter im Hangenden lagert. Teilweise in situ, teilweise umgelagert kommen die Feinsedimente in Eichenried und Eicherloh vor. Unterlagert werden sie hier von verhältnismäßig gut sortiertem, relativ kleinkalibrigem, sandarmem Schotter. Über den Feinsedimenten folgen hingegen gröbere Schotter. Im Profil Eicherloh ist der hangende Schotter durch synsedimentäre, das Feinsediment ebenfalls erfassende Kryoturbationen verwürgt.

Der an den einzelnen Fundpunkten in den Feinsedimenten festgestellte Kalkgehalt ist in Tab. 3 zusammengestellt. Er vermag bei gleicher Transportstrecke (vgl. K. Brunnacker 1959) bestimmte Hinweise zu geben; z. B. liegen die Kalkgehalte (bezogen auf das Material $<0,2 \mathrm{~mm} \phi)$ in würmeiszeitlichen und daraus hervorgegangenen spätglazialen Sedimenten etwas niedriger als in vergleichbaren postglazialen Sedimenten; außerdem gibt es eine Beziehung zwischen der Höhe des Gehaltes an "tonigen" Komponenten $(=\langle 0,002 \mathrm{~mm}$ $\phi)$ und dem Kalkgehalt. Bei den vorliegenden Proben (Tab. 3) scheint beides nicht der Fall. Der Grund dafür ist in der verschieden langen Transportstrecke zu suchen: Je weiter die Proben das Isartal abwärts entnommen sind, umso mehr nehmen die nichtkarbonatischen Komponenten zu (Eicherloh - Eichenried - Niederhummel). Im Profil Thonstetten VIII erreicht der nichtkarbonatische Anteil schließlich fast 50\%; hier handelt es sich um ein zum Ampertal gehöriges Vorkommen mit besonders langem Lauf durch das Tertiärhügelland. Diese Veränderung geht somit zum wenigsten auf pedogenetische Umformungen zurück. Der Hauptgrund ist vielmehr im damaligen paläogeographischen Bild zu 
Tabelle 3

Darstellung verschiedener Eigenschaften der beschriebenen Feinsedimente (Korngrößenzusammensetzung und Kalkgehalt nach den Untersuchungsergebnissen des bodenkundlichen Laboratoriums am Bayerischen Geologischen Landesamt München)

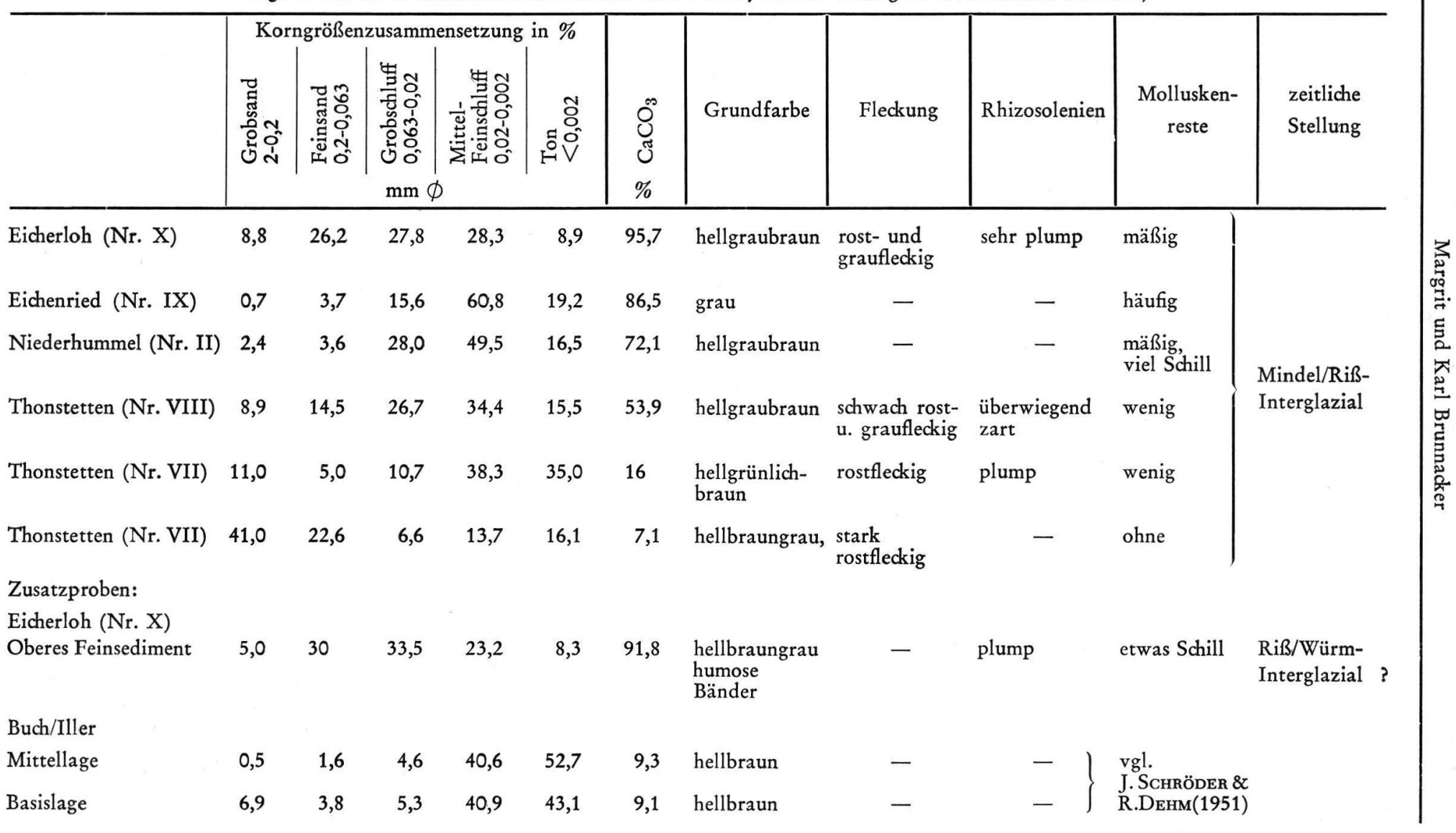


suchen: Hochgebiete aus kalkärmeren Tertiärgesteinen haben viel weiter nach Süden gereicht, als es heute der Fall ist; sie haben flußabwärts, auch während des Interglazials, in zunehmendem Umfang ihre Komponenten den Feinsedimenten beigesteuert und damit den Kalkgehalt des „reinen“ Isar- bzw. Ampermaterials verdünnt.

Eine Ausnahme scheint unter Berücksichtigung dieser Überlegung die Probe von Profil Thonstetten Nr. VII nicht zu machen; denn das Feinsediment lagert hier dem Tertiär unmittelbar auf und leitet sich weitgehend aus diesem ab.

In Tab. 3 wurden außerdem einige Zusatzproben angeführt. In Eicherloh kommt in einem höheren Niveau ein weiteres Feinsediment, das durch humose Bänder gestreift ist, vor. Es ist vom liegenden Feinsediment durch kryoturbat gestörten Schotter getrennt. Die Kalkgehalte sind praktisch die gleichen. Hingegen zeigen zwei zum Vergleich herangezogene von Buch/Iller untersuchte Proben von Feinsedimenten (s. Zusatzproben in Tab. 3), die Herr Professor Dr. H. Graul, Heidelberg, zur Verfügung stellte, die großen Unterschiede in der Körnung und im Kalkgehalt dieser molluskenführenden Ablagerungen an (vgl. J. Schröder \& R. Dehm 1951).

Die in das Mindel/Riß-Interglazial eingestuften, da an der Basis des Rißschotters lagernden Feinsedimente, sind als Schluff und Lehm, nach dem Liegenden sandiger werdend, in einer Mächtigkeit von bis $50 \mathrm{~cm}$ erhalten. Sie sind zumeist hellbraungrau gefärbt und mit kleinen grauen und rostbraunen Flecken durchsetzt, wie sie in den "TundrenNaßböden" des Lösses aufzutreten pflegen (vgl. K. BrunNACKER 1957). Beim Schlämmen der Proben haben sich außerdem im Rückstand zum Teil die als Rhizosolenien bezeichneten kalkverkitteten Wurzelröhrchen eingestellt, wie sie im Löß, aber auch in anderen geeigneten Sedimenten bei entsprechenden Bodenbildungsvorgängen vorzukommen vermögen. Wie früher gezeigt (M. Brunnacker \& K. Brunnacker 1956), besteht ein Zusammenhang $\mathrm{zwischen}$ der Form dieser Rhizosolenien und der pedogenetischen Formung des Sedimentes: typischer Löß enthält zarte,stengelige, zum Teil auch feinverzweigte kleine Rhizosolenien, durch Nässe beeinflußter Löß hingegen grobe plumpe Formen. Dies ist auch in den Feinsedimenten der Hochterrasse der Fall; denn je stärker die nässeanzeigenden grauen und rostfarbenen Flecken hervortreten, desto plumper sind die Rhizosolenien ausgebildet. Eine Ausnahme macht das Profil Eichenried; denn hier ist das Material total grau gefärbt ( $\mathrm{Gr}$ Horizont eines fossilen Gleyes), und Rhizosolenien fehlen, ebenso in Niederhummel und in einer Vergleichsprobe von Thonstetten Nr. VII.

Die Art der Naßbodenüberprägung, wie sie sich hier in Form kleiner grauer und rostfarbener Flecken anzeigt, weist zusammen mit den Rhizosolenien auf offenes Gelände, das relativ feuchten bis wechselfeuchten Bedingungen ausgesetzt war. Auffallend ist aber, daß die Fauna damit überhaupt nicht übereinstimmt. Zumindest sollten neben der Laubwaldfauna dann auch die im Grasland gehäuft vorkommenden Kleinschnecken in größerer Arten- und Individuenzahl zu erwarten sein.

Damit ist folgende Möglichkeit nicht von der Hand zu weisen: Die Laubwaldfauna wurde, soweit sie nicht im Sedimentationsraum lebte, in die Feinsedimente auf nur kurze Entfernung aus Auewaldbereichen eingeschwemmt und zwar im Rahmen eines kurzfristigen Sedimentationsvorganges. In einer späteren Phase kam es zu einer Lichtung der Wälder und zu einer pedogenetischen Überformung der Feinsedimente durch Nässe. Und hier wäre es nun denkbar, daß die "Jüngere", bereits recht kühles oder sogar kaltes Klima anzeigende Fauna des Hochterrassenschotters (vgl. R. SCHRöDF,R 1915) gleichaltrig mit dieser pedogenetischen Veränderung der Feinsedimente ist. Dann muß aber ein von Laubwaldklima zu subarktischem Klima überleitender Abschnitt dazwischengelegen haben. Dieser läßt sich durch zwischengeschaltete Schotter im Profil Ismaning fassen (vgl. R. SCHRöDER 1915); andernorts war diese Zeit vielleicht durch eine terrestre Bodenbildung vertreten, im Profil Eichenried vielleicht auch durch die genannte totale Vergleyung, und 
Tabelle 4

Deutungsversuch des Sedimentationsablaufes von der Mindeleiszeit bis zur Rißeiszeit im Nordosten von München

Ablagerung des fluvioglazialen Hochterrassenschotters, z. T. mit synsedimentären Kryoturbationen (z. B. in Eicherloh)

Hochglazial

der Rißeiszeit

Naßboden - Überprägung der noch an der OberKühle bis kalte

fläche liegenden Fein- Molluskenfauna

Beginn ? der

sedimente

Terrestre Bodenbildung

Gley in

(nicht erhalten)

Eichenried

Liegendfazies des Hochterrassenschotters (in Niederhummel mit sehr viel

Sedimentationslücke Tertiärmaterial), z.T. mit aufgearbeiteten Feinsedimenten

Rißeiszeit

bis

ausgehendes Mindel/Riß-Inter-

glazial

Mindel/Riß-

Feinsedimente mit Laubwald-Molluskenfauna, z. T. relativ feinkörnigen Interglazial

Kalkschotter überlagernd

grober Kalkschotter in Eicherloh

Mindeleiszeit

im Profil Niederhummel fehlen die Rhizosolenien möglicherweise deshalb, weil hier das Feinsediment vor dieser Umwandlung aufgearbeitet und einsedimentiert wurde. ${ }^{1}$ )

Im Profil Eichenried sind Schotter und Randpartien des (z. T. aufgearbeiteten) Lehms rostbraun verfärbt. Hierbei handelt es sich um sekundäre Überprägungen durch einen fossilen Go-Horizont des Riß/Würm-Interglazials. Diese Imprägnationen stehen also in keinem direkten und genetischen Zusammenhang mit der erwähnten Naßboden-Überprägung der Feinsedimente, die in die vorangegangene Eiszeit gehört.

Damit kann die diskutierte Abfolge gemäß der in Tab. 4 dargestellten Form gegliedert werden. Es könnte weiter überlegt werden, ob durch die Jüngere Fauna des Hochterrassenschotters vielleicht ein in die Rißeiszeit eingeschaltetes Interstadial angezeigt wird. Doch reicht dafür das bisher bekannt gewordene Material keinesfalls aus, zumal schon die in Tab. 4 angeführte Möglichkeit weiterer Untermauerung bedarf.

Sicher ist hingegen, daß im Profil Eicherloh im tieferen Untergrund nicht ein Aquivalent des Deckenschotters im Sinne von A. PENCK vorliegt, sondern vielmehr ein fluvioglazialer Grobschotter der Mindeleiszeit, die J. KNAUER (1938) südlich München unter rißeiszeitlichen Ablagerungen nachzuweisen vermochte.

1) Der die "Jüngere Fauna“ überdeckende Hochterrassenschotter ist den Beschreibungen von O. M. REIS (1922) zufolge dort synsedimentär kryoturbat gestört, wo er als eine nur verhältnismäßig geringmächtige Decke entwickelt ist. Da entsprechende Kryoturbationen auch im Hochterrassenschotter in Profil Eicherloh - jedoch die Feinsedimente mit der interglazialen Fauna überprägend - vorkommen, spricht auch dieser Befund für die vorgetragene, in Tab. 4 dargestellte Deutung hinsichtlich einer z.T. vorhandenen Sedimentationslücke zwischen dem interglazialen Feinsediment und der "Jüngeren Fauna". 


\section{Schrift um}

Brunnacker, K.: Die Geschichte der Böden im jüngeren Pleistozän in Bayern. - Geol. Bavarica 34, München 1957. - - Geol. Karte von Bayern 1:25000 Nr. 7636 Freising Süd mit Erläut. - München 1959.

Brunnacker, M. \& Brunnacker, K.: Die Molluskenfauna einiger Lößprofile im Donautal. - Geol. Bl. NO-Bayern 6, S. 96-106, Erlangen 1956.

Ebers, E.: Drumlinkerne, ältere Würmschotter und das Würm-Interstadial-Profil von Hörmating/ Obb. - Eiszeitalter u. Gegenwart 11, S. 64-76, Öhringen 1960.

Knauer, J.: Die Mindel-Eiszeit, die Zeit größter Vergletscherung in Süddeutschland. - Abh. geol. Landesunters. bayer. Oberbergamt 29, S. 35-45, München 1938.

Kursch, M.: Zur Geologie der Münchener Schotterfläche rechts der Isar, nördlich von Ismaning. Dipl.Arbeit TH München, München 1962.

Nathan, H.: Ein interglacialer Schotter südlich Moosburg in Oberbayern mit Fagotia acicularis FÉrussaC (Melanopsenkies). - Geol. Bavarica 19, S. 315-334, München 1953.

ReIs, O. M.: Ưber einzelne Beobachtungen im Diluvium der Umgebung von München. - Geognost. Jh. 34, S. 143-168, München 1922.

SchröDer, J. \& Dehm, R.: Die Molluskenfauna aus der Lehm-Zwischenlage des Deckenschotters von Fischach, Kreis Augsburg. - Geol. Bavarica 6, S. 118-120, München 1951.

SchröDER, R.: Die Conchylien des Münchner Gebiets vom Pleistocaen bis zur Gegenwart. - Nachr.blatt dt. malakozoolog. Ges., H. 3 u. 4, 1915.

Manuskr. eingeg. 30. 3. 1962.

Anschrift der Verf.: Dr. Margrit Brunnacker und Dr. Karl Brunnacker, München 27, Möhlstr. 12a. 